\title{
Surface-circulation change in the southwest Pacific Ocean across the Middle Eocene Climatic Optimum: inferences from dinoflagellate cysts and biomarker paleothermometry
}

Margot J. Cramwinckel et al.

Correspondence to: Margot J. Cramwinckel (m.j.cramwinckel@ soton.ac.uk)

The copyright of individual parts of the supplement might differ from the CC BY 4.0 License. 


\section{Supplementary Figures}

(a)

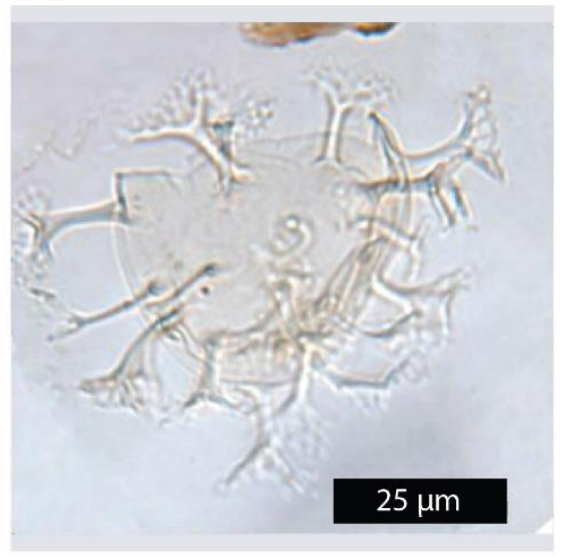

(d)

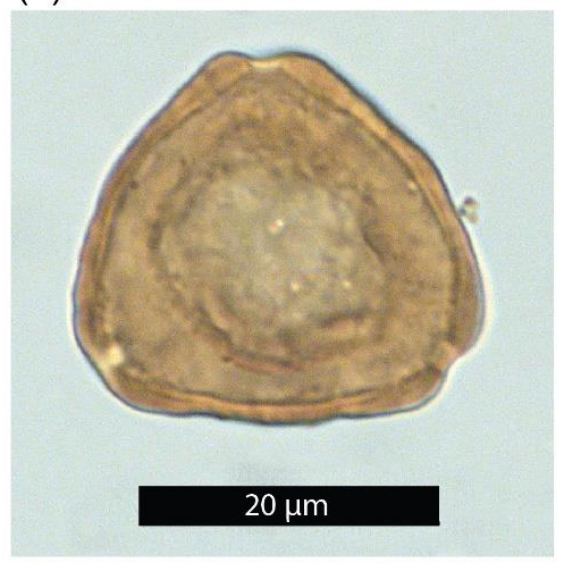

(b)

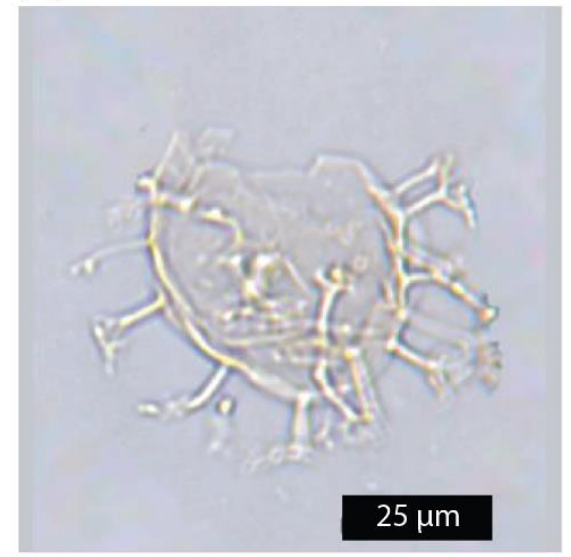

(e)

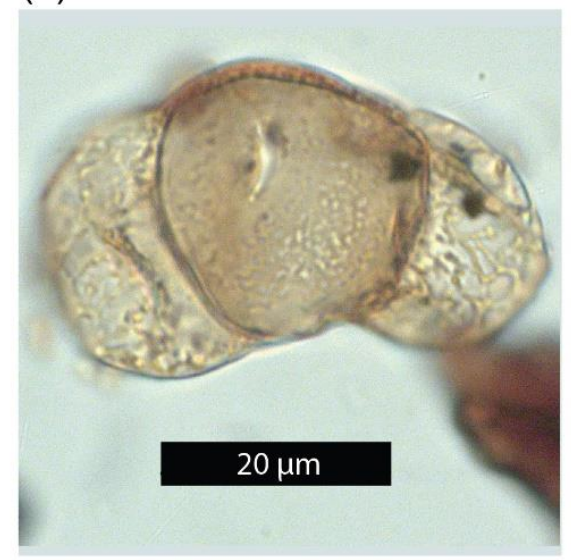

(c)

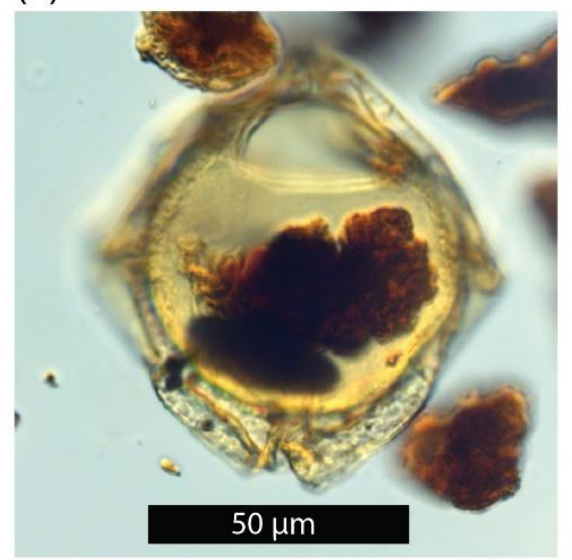

(f)

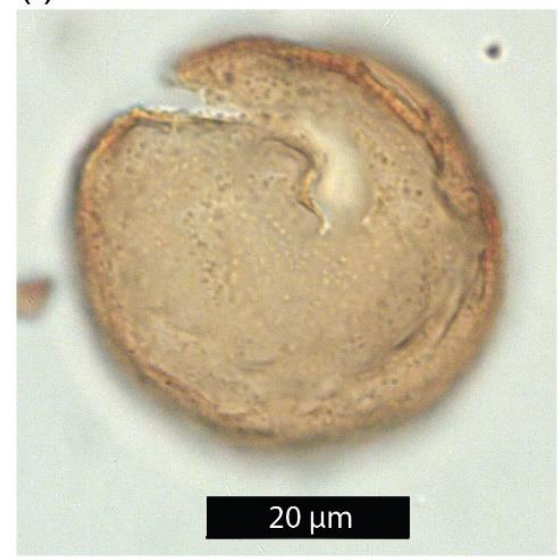

Supplementary Figure 1. Plate with light microscope images of relevant palynomorphs encountered in this study. (a) Dinocyst Enneadocysta dictyostila from sample 1170D 21R 4W 85-87 cm (EFC unavailable), scale bar $25 \mu \mathrm{m}$. (b) Dinocyst Enneadocysta multicornuta from sample 1170D 23R 2W 85-87 cm (EFC unavailable), scale bar 25 $\mu \mathrm{m}$. (c) Dinocyst Dracodinium rhomboideum from sample L86 slide 2 (EFC E48.1), scale bar $50 \mu \mathrm{m}$. (d) Pollen Myricipites harrisii from sample L85 slide 1 (EFC J15.4), scale bar $20 \mu \mathrm{m}$. (e) Pollen Podocarpidites ellipticus from sample L84 slide 1 (EFC G19.2), scale bar $20 \mu \mathrm{m}$. (f) Pollen Dilwynites granulatus from sample L87 slide 1 (EFC J16.1), scale bar $20 \mu \mathrm{m}$. 


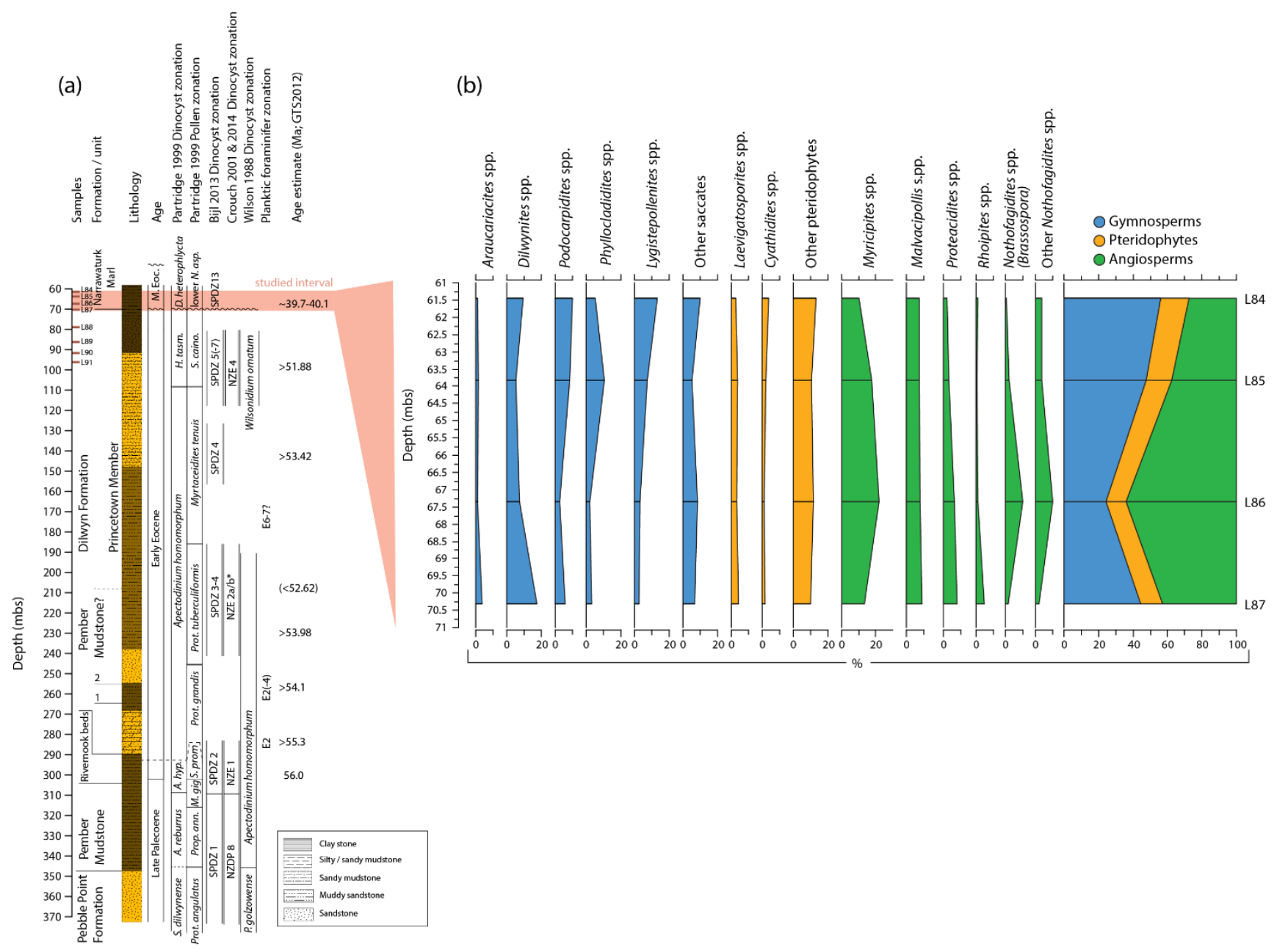

Supplementary Figure 2. (a) Stratigraphy of the Latrobe-1 borehole, including studied samples, lithologic units, lithology and age constraints based on microfossil biostratigraphy. The interval analysed in this study is highlighted in pink. Figure adapted from (Frieling et al., 2018a). (b) Relative abundances of representative sporomorph taxa in samples L84-L87 from the Latrobe-1 borehole, in percentage of total sporomorph assemblage. 
(a) NMDS Site 1170 (all taxa shown)
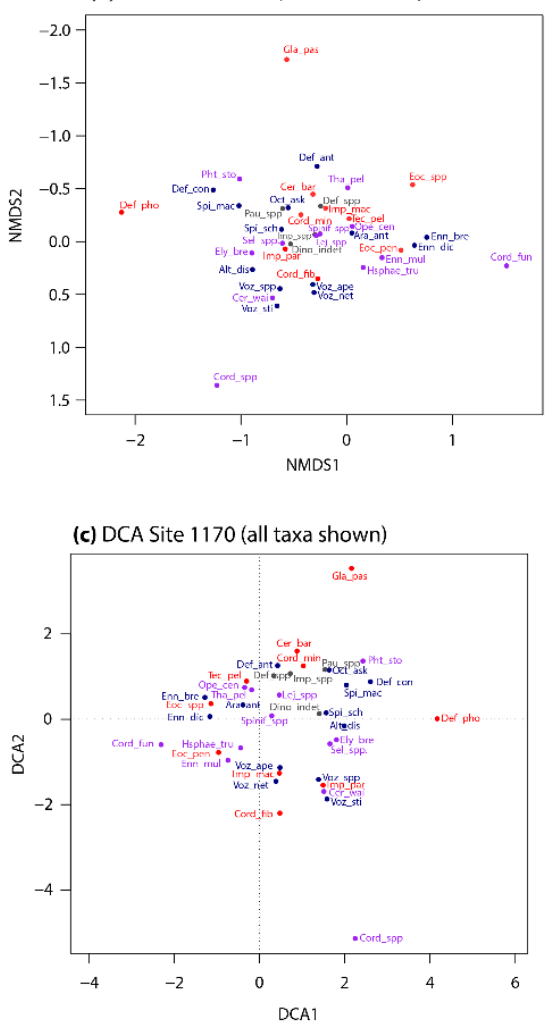

(e) Site 1170

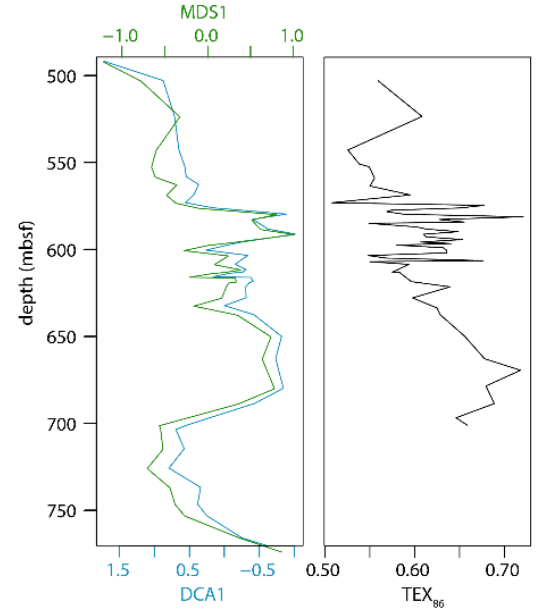

(b) NMDS Site 1172 (all taxa shown)

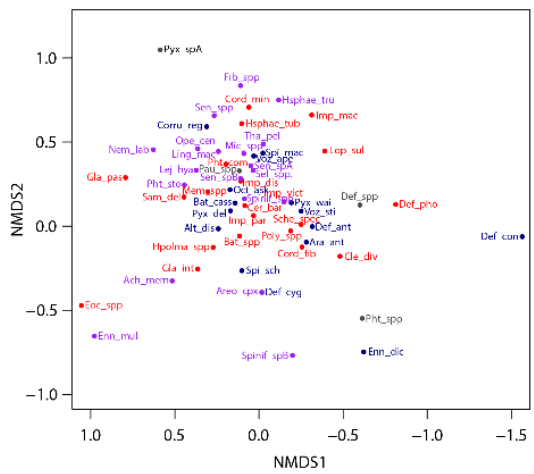

(d) DCA Site 1172 (all taxa shown)

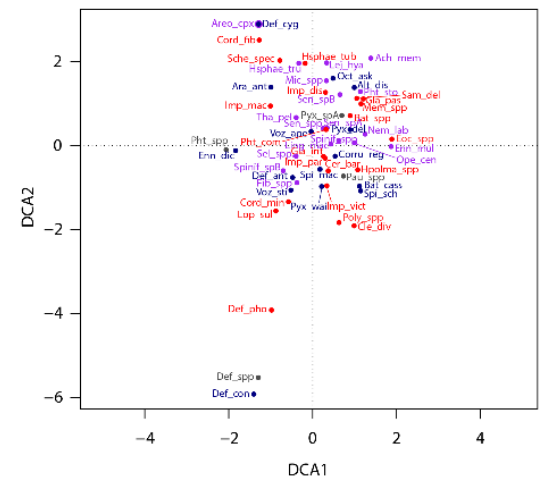

(f) Site 1172

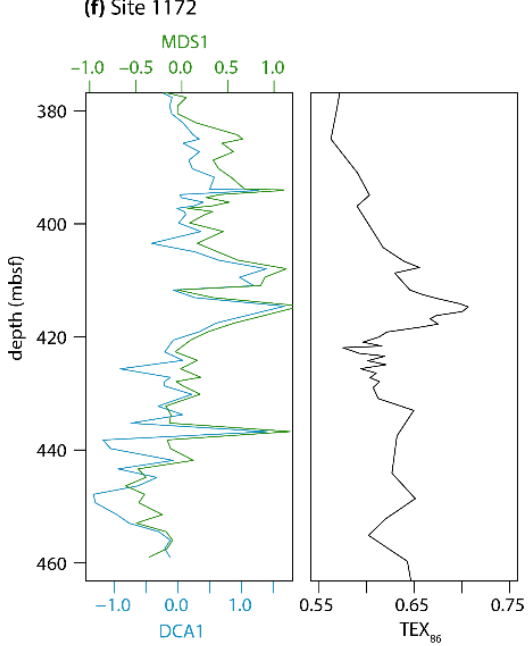

Supplementary Figure 3. Additional ordination results. Nonmetric multidimensional scaling (NMDS) ordination diagram for the dinocyst assemblage data of Site 1170 (a) and Site 1172 (b). Detrended correspondence analysis (DCA) ordination diagram for the dinocyst assemblage data of Site 1170 (c) and Site 1172 (d). Species scores in a-d as circles, colour-coded by biogeographic affinity (red, mid-low latitude; purple, cosmopolitan; blue, endemic; grey, not assigned). Full names for dinocyst abbreviations can be found in the Supplementary Datafile. First axis of DCA (blue) and NMDS (green) analysis of Site 1170 (e) and Site 1172 (f), together with the respective TEX 86 records (black). 

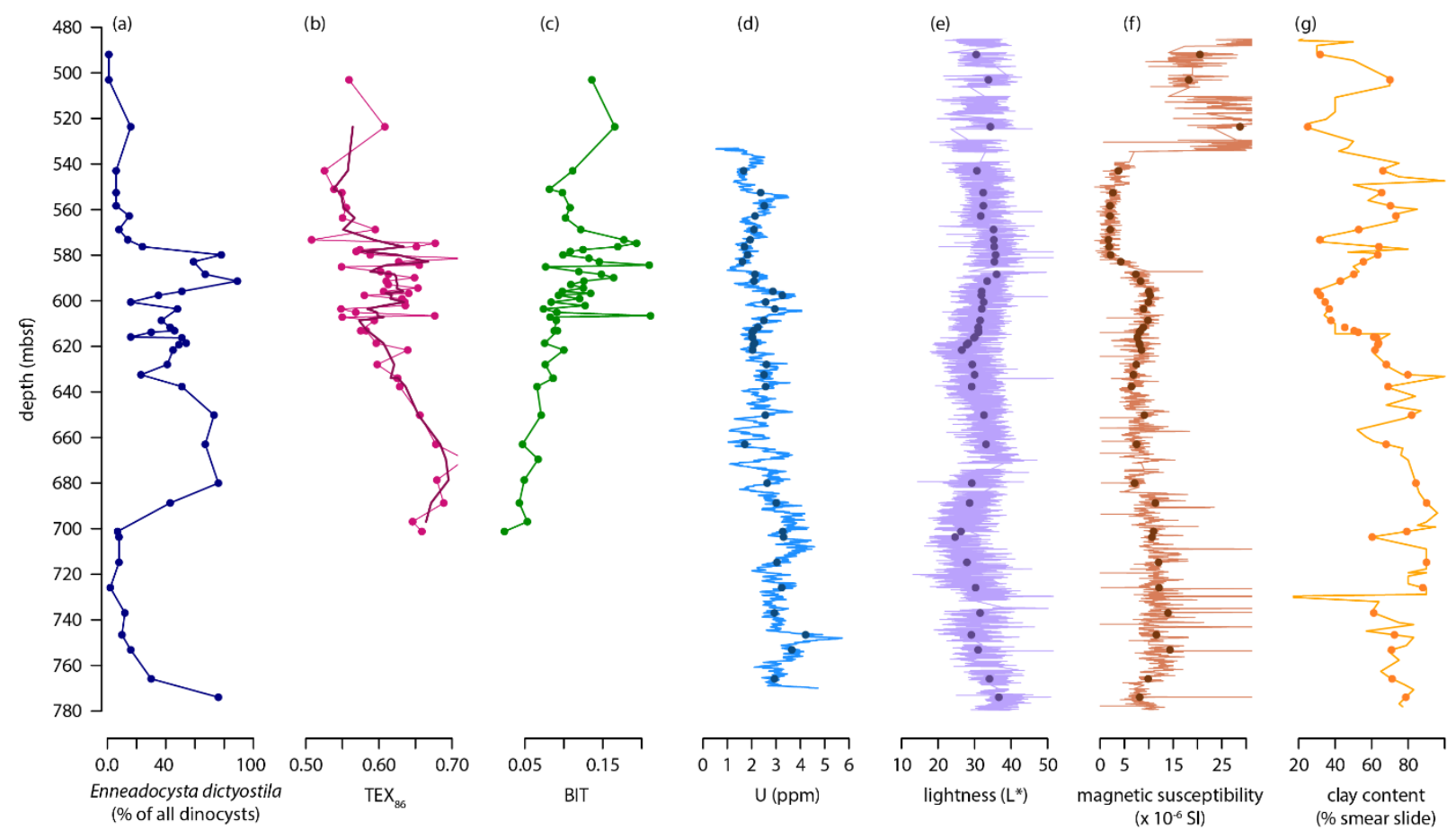

Supplementary Figure 4. Environmental proxy records over the MECO interval of Site 1170, as used in CCA analysis (b-g). Original data plotted as line, data interpolated to depth of dinocyst samples plotted as dots. Plotted against depth in metres below sea level. (a) Relative abundance of Enneadocysta dictyostila (percentage of total dinocyst assemblage; dark blue dots and line). (b) $\mathrm{TEX}_{86}$ (pink dots and line), with three-point moving average (purple lines). (c) BIT (green dots and line). (d) Sedimentary uranium content (ppm; blue dots and line). (e) Spectrophotometric lightness (CIELAB L*; purple dots and line). (f). Core-measured magnetic susceptibility ( $\mathrm{x}$ $\left.10^{-6} \mathrm{SI}\right)$. (g). Clay content (\% of smear slide). Data in panels d-g from Exon et al. (2001). 

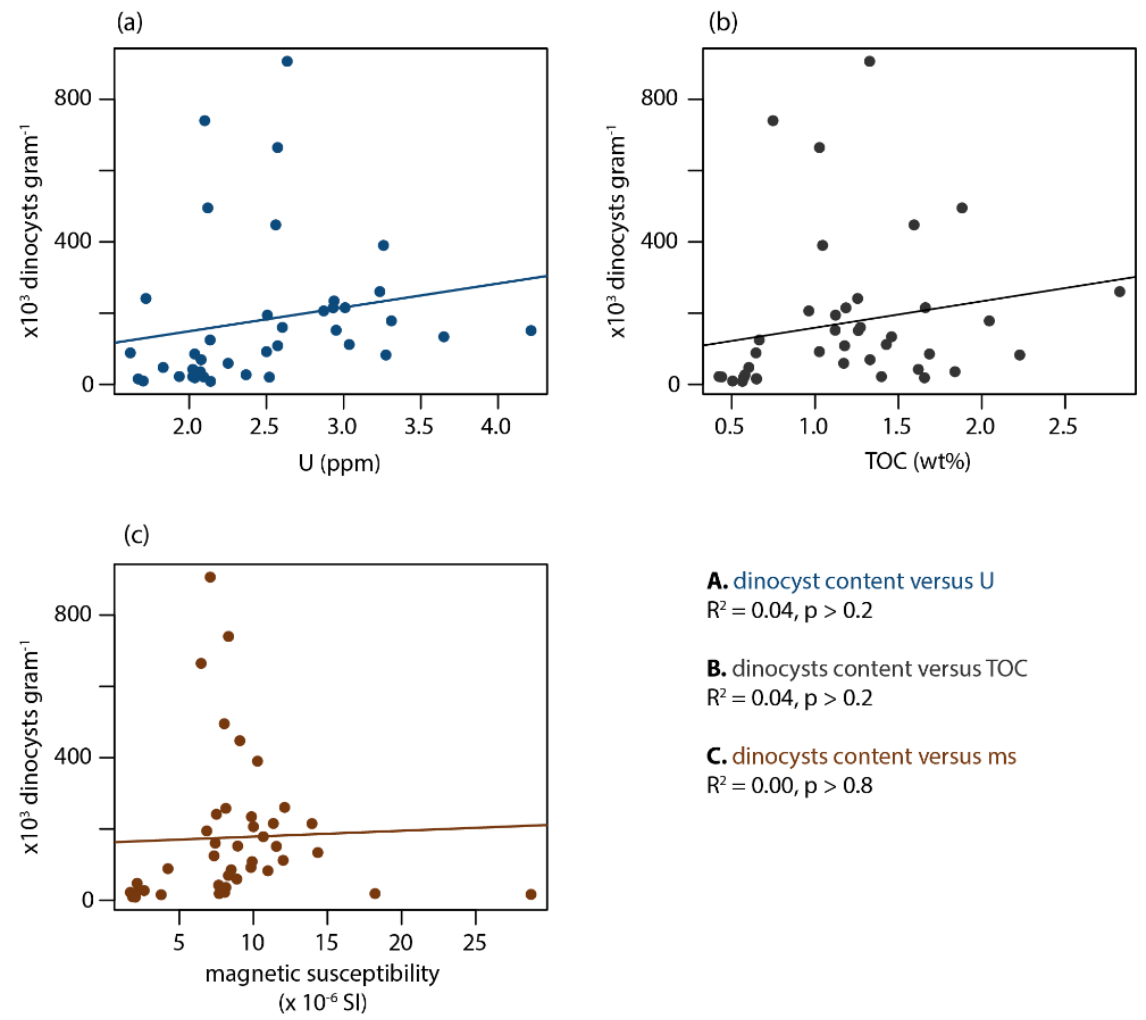

A. dinocyst content versus $U$

$\mathrm{R}^{2}=0.04, \mathrm{p}>0.2$

B. dinocysts content versus TOC

$R^{2}=0.04, p>0.2$

C. dinocysts content versus ms

$\mathrm{R}^{2}=0.00, \mathrm{p}>0.8$

Supplementary Figure 5. Scatter plots and regression analysis of sedimentary dinocyst content as a function of selected proxy records of Site 1170, indicating no significant correlation. (a) Dinoflagellate cyst content (cysts per gram of dry sediment) against uranium content (ppm). (b) Dinoflagellate cyst content (cysts per gram of dry sediment) against total organic carbon content (weight percentage). (c) Dinoflagellate cyst content (cysts per gram of dry sediment) against magnetic susceptibility (x 10 $0^{-6} \mathrm{SI}$ ). U, TOC and ms data from Exon et al. (2001). 
Supplementary Table 1. List of observed dinoflagellate cyst species, including their biogeographic affinitiy as used in the present study (bg1), an alternative biogeographic grouping (bg2), cyst type, and at which of the studied sites (Site 1170, Site 1172, Latrobe-1 borehole, Hampden Beach section) they were encountered. Abbreviations: g, gonyaulacoid; p, peridinioid; m, mid-/low-latitude; c, cosmopolitan; b, bipolar; tf, endemic "Transantarctic Flora"; e, endemic Southern Ocean; o, other (unknown or ambivalent); wetz, wetzeliellioids (here considered cosmopolitan, could also be considered mid-/low-latitude based on their warm affinity); gonio, goniodomids.

\begin{tabular}{|c|c|c|c|c|c|c|c|c|}
\hline dinocyst taxon & abbreviation & bg1 & bg2 & cyst & 1170 & 1172 & Latrobe & Hampden \\
\hline Achilleodinium biformoides & Ach_bi & $\mathrm{c}$ & $\mathrm{c}$ & $\mathrm{g}$ & & & Latrobe & \\
\hline $\begin{array}{l}\text { Achomosphaera } \\
\text { membraniphora }\end{array}$ & Ach_mem & $\mathrm{c}$ & $\mathrm{c}$ & $\mathrm{g}$ & & 1172 & & \\
\hline Achomosphaera ramulifera & Ach_ram & $\mathrm{c}$ & $\mathrm{c}$ & $\mathrm{g}$ & & 1172 & & \\
\hline Achomosphaera spp. (pars) & Ach_spp & $\mathrm{c}$ & $\mathrm{c}$ & $\mathrm{g}$ & & & Latrobe & Hampden \\
\hline Adnatosphaeridium spp. & Ad_spp & $\mathrm{c}$ & $\mathrm{c}$ & $\mathrm{g}$ & & & Latrobe & \\
\hline Alisocysta circumtabulata & Ali_cir & $\mathrm{c}$ & $\mathrm{c}$ & $\mathrm{g}$ & & & & Hampden \\
\hline Alterbidinium acutulum & Alt_acu & $\mathrm{e}$ & $\mathrm{e}$ & $\mathrm{p}$ & & 1172 & & \\
\hline Alterbidinium distinctum & Alt_dis & $\mathrm{tf}$ & tf & $\mathrm{p}$ & 1170 & 1172 & & \\
\hline $\begin{array}{l}\text { Apectodinium } \\
\text { homomorphum }\end{array}$ & Ape_hom & wetz & $\mathrm{m}$ & $\mathrm{p}$ & & 1172 & & \\
\hline Apteodinium australiense & Apt_aus & $\mathrm{m}$ & $\mathrm{m}$ & $\mathrm{g}$ & & 1172 & & \\
\hline Arachnodinium antarcticum & Ara_ant & $\mathrm{tf}$ & $\mathrm{tf}$ & $\mathrm{g}$ & 1170 & 1172 & & \\
\hline Areoligera Сpx. (pars) & Areo_cpx & $\mathrm{c}$ & $\mathrm{c}$ & $\mathrm{g}$ & & 1172 & Latrobe & \\
\hline Batiacasphaera cassicula & Bat_cass & $\mathrm{e}$ & $\mathrm{e}$ & $\mathrm{g}$ & & 1172 & & Hampden \\
\hline Batiacasphaera spp. & Bat_spp & $\mathrm{m}$ & $\mathrm{m}$ & $\mathrm{g}$ & & 1172 & Latrobe & \\
\hline Brigantedinium spp. & Brig_spp & $\mathrm{c}$ & $\mathrm{c}$ & $\mathrm{p}$ & & 1172 & & \\
\hline Cerebrocysta bartonensis & Cer_bar & $\mathrm{m}$ & $\mathrm{m}$ & $\mathrm{g}$ & 1170 & 1172 & & \\
\hline Cerebrocysta spp. (pars) & Cer_spp & $\mathrm{c}$ & $\mathrm{c}$ & $\mathrm{g}$ & & 1172 & & \\
\hline Cerebrocysta waipawaensis & Cer_wai & $\mathrm{c}$ & $\mathrm{c}$ & $\mathrm{g}$ & 1170 & & & Hampden \\
\hline $\begin{array}{l}\text { Charlesdowniea } \\
\text { coleothrypta }\end{array}$ & Char_col & wetz & $\mathrm{c}$ & $\mathrm{p}$ & & 1172 & & \\
\hline $\begin{array}{l}\text { Cleistosphaeridium } \\
\text { diversispinosum }\end{array}$ & Cle_div & $\mathrm{m}$ & $\mathrm{m}$ & $\mathrm{g}$ & & 1172 & & \\
\hline $\begin{array}{l}\text { Cleistosphaeridium } \\
\text { placacanthum }\end{array}$ & Cle_pla & $\mathrm{m}$ & $\mathrm{m}$ & $\mathrm{g}$ & & 1172 & & \\
\hline $\begin{array}{l}\text { Cleistosphaeridium spp. } \\
\text { (pars) }\end{array}$ & Cle_spp & $\mathrm{c}$ & $\mathrm{c}$ & $\mathrm{g}$ & & & Latrobe & Hampden \\
\hline $\begin{array}{l}\text { Cordosphaeridium Cpx. } \\
\text { (pars) }\end{array}$ & Cord_spp & $\mathrm{c}$ & $\mathrm{c}$ & $\mathrm{g}$ & 1170 & & Latrobe & \\
\hline $\begin{array}{l}\text { Cordosphaeridium } \\
\text { fibrospinosum }\end{array}$ & Cord_fib & $\mathrm{m}$ & $\mathrm{m}$ & $\mathrm{g}$ & 1170 & 1172 & Latrobe & Hampden \\
\hline $\begin{array}{l}\text { Cordosphaeridium } \\
\text { funiculatum }\end{array}$ & Cord_fun & $\mathrm{c}$ & $\mathrm{c}$ & $\mathrm{g}$ & 1170 & & & \\
\hline Cordosphaeridium minimum & Cord_min & $\mathrm{m}$ & $\mathrm{m}$ & $\mathrm{g}$ & 1170 & 1172 & & \\
\hline Corrudinium incompositum & Cor_inc & $\mathrm{m}$ & $\mathrm{m}$ & $\mathrm{g}$ & 1170 & & Latrobe & \\
\hline Corrudinium regulare & Corru_reg & $\mathrm{tf}$ & $\mathrm{tf}$ & $\mathrm{g}$ & & 1172 & & \\
\hline Corrudinium spp. (pars) & Corru_spp & $\mathrm{o}$ & $\mathrm{O}$ & $\mathrm{g}$ & & & Latrobe & \\
\hline Cribroperidinium spp. (pars) & Cri_spp & $\mathrm{c}$ & $\mathrm{c}$ & $\mathrm{g}$ & & 1172 & & \\
\hline
\end{tabular}




\begin{tabular}{|c|c|c|c|c|c|c|c|c|}
\hline $\begin{array}{l}\text { Cribroperidinium } \\
\text { tenuitabulatum }\end{array}$ & Cri_ten & $\mathrm{c}$ & $\mathrm{c}$ & $\mathrm{g}$ & & & & Hampden \\
\hline Danea crassimurata & Dan_cra & $\mathrm{c}$ & $\mathrm{c}$ & $\mathrm{g}$ & & & & Hampden \\
\hline Dapsilidinium spp. & Daps_spp & $\mathrm{m}$ & $\mathrm{m}$ & $\mathrm{g}$ & & 1172 & Latrobe & Hampden \\
\hline Deflandrea antarctica & Def_ant & $\mathrm{tf}$ & tf & $\mathrm{p}$ & 1170 & 1172 & & Hampden \\
\hline Deflandrea convexa & Def_con & $\mathrm{tf}$ & $\mathrm{tf}$ & $\mathrm{p}$ & 1170 & 1172 & & \\
\hline Deflandrea cygniformis & Def_cyg & $\mathrm{tf}$ & $\mathrm{tf}$ & $\mathrm{p}$ & 1170 & 1172 & & \\
\hline Deflandrea phosphoritica & Def_pho & $\mathrm{m}$ & $\mathrm{m}$ & $\mathrm{p}$ & 1170 & 1172 & Latrobe & \\
\hline $\begin{array}{l}\text { Deflandrea sp. A cf. } \\
\text { Brinkhuis } 2003\end{array}$ & Def_spA & $\mathrm{tf}$ & $\mathrm{tf}$ & $\mathrm{p}$ & & 1172 & & \\
\hline Deflandrea spp. (pars) & Def_spp & o & o & $\mathrm{p}$ & 1170 & 1172 & Latrobe & \\
\hline Diphyes colligerum & Diph_col & $\mathrm{c}$ & $\mathrm{c}$ & $\mathrm{g}$ & & 1172 & & Hampden \\
\hline Diphyes spp. (pars) & Diph_spp & $\mathrm{c}$ & $\mathrm{c}$ & $\mathrm{g}$ & & & Latrobe & \\
\hline Distatodinium ellipticum & Dis_ell & $\mathrm{m}$ & $\mathrm{m}$ & $\mathrm{g}$ & & & Latrobe & \\
\hline Distatodinium paradoxum & Dis_par & $\mathrm{m}$ & $\mathrm{m}$ & $\mathrm{g}$ & & 1172 & Latrobe & \\
\hline Dracodinium rhomboideum & Dra_rho & wetz & $\mathrm{c}$ & $\mathrm{p}$ & & & Latrobe & \\
\hline Elytrocysta brevis & Ely_bre & $\mathrm{c}$ & $\mathrm{c}$ & $\mathrm{g}$ & 1170 & & & Hampden \\
\hline Enneadocysta brevistila & Enn_bre & $\mathrm{tf}$ & $\mathrm{tf}$ & $\mathrm{g}$ & 1170 & & & \\
\hline Enneadocysta dictyostila & Enn_dic & $\mathrm{tf}$ & tf & $\mathrm{g}$ & 1170 & 1172 & & Hampden \\
\hline Enneadocysta multicornuta & Enn_mul & $\mathrm{c}$ & $\mathrm{c}$ & $\mathrm{g}$ & 1170 & 1172 & Latrobe & \\
\hline Enneadocysta pectiniformis & Enn_pec & $\mathrm{m}$ & $\mathrm{m}$ & $\mathrm{g}$ & & & Latrobe & \\
\hline Enneadocysta spp. (pars) & Enn_spp & $\mathrm{o}$ & o & $\mathrm{g}$ & & & Latrobe & \\
\hline $\begin{array}{l}\text { Enneadocysta- } \\
\text { Oligosphaeridum } \\
\text { intermediate }\end{array}$ & Enn_Oli & o & o & $\mathrm{g}$ & & & Latrobe & \\
\hline Eocladopyxis peniculata & Eoc_pen & gonio & $\mathrm{m}$ & $\mathrm{g}$ & 1170 & & & \\
\hline Eocladopyxis spp. (pars) & Eoc_spp & gonio & $\mathrm{m}$ & $\mathrm{g}$ & 1170 & 1172 & Latrobe & Hampden \\
\hline Fibrocysta spp. & Fib_spp & $\mathrm{c}$ & $\mathrm{c}$ & $\mathrm{g}$ & & 1172 & & Hampden \\
\hline Gelatia inflata & Gel_inf & $\mathrm{b}$ & $\mathrm{b}$ & $\mathrm{g}$ & & 1172 & & \\
\hline Glaphyrocysta delicata & Gla_del & $\mathrm{m}$ & $\mathrm{m}$ & $\mathrm{g}$ & & 1172 & & Hampden \\
\hline Glaphyrocysta intricata & Gla_int & $\mathrm{m}$ & $\mathrm{m}$ & $\mathrm{g}$ & & 1172 & & \\
\hline Glaphyrocysta pastielsii & Gla_pas & $\mathrm{m}$ & $\mathrm{m}$ & $\mathrm{g}$ & 1170 & 1172 & & \\
\hline Glaphyrocysta retiintexta & Gla_ret & $\mathrm{m}$ & $\mathrm{m}$ & $\mathrm{g}$ & & 1172 & & \\
\hline $\begin{array}{l}\text { Hemiplacophora } \\
\text { semilunifera }\end{array}$ & Hem_sem & $\mathrm{c}$ & $\mathrm{c}$ & $\mathrm{g}$ & & & Latrobe & \\
\hline Heteraulacacysta spp. & Het_spp & gonio & $\mathrm{c}$ & $\mathrm{g}$ & & & Latrobe & Hampden \\
\hline Homotryblium spp. & Hom_spp & gonio & $\mathrm{c}$ & $\mathrm{g}$ & 1170 & & & \\
\hline $\begin{array}{l}\text { Hystiocysta / Elytrocysta } \\
\text { spp. (pars) }\end{array}$ & Hcyst_Ely & $\mathrm{c}$ & $\mathrm{c}$ & $\mathrm{g}$ & & 1172 & Latrobe & Hampden \\
\hline Hystrichokolpoma rigaudiae & Hpolma_rig & $\mathrm{m}$ & $\mathrm{m}$ & $\mathrm{g}$ & & 1172 & & Hampden \\
\hline Hystrichokolpoma spinosum & Hpoma_spi & $\mathrm{m}$ & $\mathrm{m}$ & g & & 1172 & & \\
\hline $\begin{array}{l}\text { Hystrichokolpoma spp. } \\
\text { (pars) }\end{array}$ & Hpolma_spp & $\mathrm{m}$ & $\mathrm{m}$ & $\mathrm{g}$ & & 1172 & Latrobe & \\
\hline $\begin{array}{l}\text { Hystrichokolpoma } \\
\text { truncatum }\end{array}$ & Hpolma_tru & $\mathrm{m}$ & $\mathrm{m}$ & $\mathrm{g}$ & & 1172 & & \\
\hline $\begin{array}{l}\text { Hystrichosphaeridium } \\
\text { truswelliae }\end{array}$ & Hsphae_tru & $\mathrm{c}$ & $\mathrm{c}$ & $\mathrm{g}$ & 1170 & 1172 & & \\
\hline
\end{tabular}




\begin{tabular}{|c|c|c|c|c|c|c|c|c|}
\hline $\begin{array}{l}\text { Hystrichosphaeridium } \\
\text { tubiferum }\end{array}$ & Hsphae_tub & $\mathrm{m}$ & $\mathrm{m}$ & $\mathrm{g}$ & & 1172 & Latrobe & Hampden \\
\hline Impagidinium dispertitum & Imp_dis & $\mathrm{m}$ & $\mathrm{m}$ & $\mathrm{g}$ & & 1172 & & Hampden \\
\hline Impagidinium maculatum & Imp_mac & $\mathrm{m}$ & $\mathrm{m}$ & $\mathrm{g}$ & 1170 & 1172 & & Hampden \\
\hline $\begin{array}{l}\text { Impagidinium } \\
\text { parvireticulatum }\end{array}$ & Imp_par & $\mathrm{m}$ & $\mathrm{m}$ & $\mathrm{g}$ & 1170 & 1172 & & \\
\hline Impagidinium spp. (pars) & Imp_spp & o & o & $\mathrm{g}$ & 1170 & 1172 & Latrobe & \\
\hline Impagidinium victorianum & Imp_vict & $\mathrm{m}$ & $\mathrm{m}$ & $\mathrm{g}$ & & 1172 & & \\
\hline Kallosphaeridium spp. & Kal_spp & $\mathrm{c}$ & $\mathrm{c}$ & $\mathrm{g}$ & & 1172 & Latrobe & \\
\hline Lejeunecysta hyalina & Lej_hya & $\mathrm{c}$ & $\mathrm{c}$ & $\mathrm{p}$ & & 1172 & & \\
\hline Lejeunecysta spp. (pars) & Lej_spp & $\mathrm{c}$ & $\mathrm{c}$ & $\mathrm{p}$ & 1170 & & Latrobe & Hampden \\
\hline $\begin{array}{l}\text { Lingulodinium } \\
\text { machaerophorum }\end{array}$ & Ling_mac & $\mathrm{c}$ & $\mathrm{c}$ & $\mathrm{g}$ & & 1172 & & \\
\hline Lingulodinium spp. (pars) & Ling_spp & $\mathrm{c}$ & $\mathrm{c}$ & $\mathrm{g}$ & & & Latrobe & \\
\hline Lophocysta sulcolimbata & Lop_sul & $\mathrm{m}$ & $\mathrm{m}$ & $\mathrm{g}$ & 1170 & 1172 & & \\
\hline $\begin{array}{l}\text { Melitasphaeridium } \\
\text { pseudorecurvatum }\end{array}$ & Mel_pseu & $\mathrm{c}$ & $\mathrm{c}$ & $\mathrm{g}$ & & & Latrobe & Hampden \\
\hline Membranosphaera spp. & Mem_spp & $\mathrm{m}$ & $\mathrm{m}$ & $\mathrm{g}$ & & 1172 & & \\
\hline Microdinium spp. & Mic_spp & $\mathrm{c}$ & $\mathrm{c}$ & $\mathrm{g}$ & & 1172 & & \\
\hline $\begin{array}{l}\text { Nematosphaeropsis } \\
\text { labyrinthus }\end{array}$ & Nem_lab & $\mathrm{c}$ & $\mathrm{c}$ & $\mathrm{g}$ & & 1172 & & \\
\hline Octodinium askiniae & Oct_ask & tf & tf & $\mathrm{p}$ & 1170 & 1172 & & Hampden \\
\hline $\begin{array}{l}\text { Operculodinium } \\
\text { centrocarpum }\end{array}$ & Ope_cen & $\mathrm{c}$ & $\mathrm{c}$ & $\mathrm{g}$ & 1170 & 1172 & & \\
\hline Operculodinium spp. (pars) & Oper_spp & $\mathrm{c}$ & $\mathrm{c}$ & $\mathrm{g}$ & & 1172 & Latrobe & Hampden \\
\hline Paucispaeridium spp. & Pau_spp & o & o & o & 1170 & 1172 & & Hampden \\
\hline Pentadinium spp. & Pen_spp & $\mathrm{c}$ & $\mathrm{c}$ & $\mathrm{g}$ & & & Latrobe & \\
\hline $\begin{array}{l}\text { Phthanoperidinium } \\
\text { comatum ** }\end{array}$ & Pht_com & $\mathrm{c}$ & $\mathrm{c}$ & $\mathrm{p}$ & & 1172 & Latrobe & \\
\hline $\begin{array}{l}\text { Phthanoperidinium spp. } \\
\text { (pars) }\end{array}$ & Pht_spp & o & $\mathrm{o}$ & $\mathrm{p}$ & & 1172 & Latrobe & \\
\hline $\begin{array}{l}\text { Phthanoperidinium } \\
\text { stockmansii** }\end{array}$ & Pht_sto & $\mathrm{c}$ & $\mathrm{c}$ & $\mathrm{p}$ & 1170 & 1172 & & Hampden \\
\hline Polysphaeridium spp. & Poly_spp & gonio & $\mathrm{m}$ & $\mathrm{g}$ & & 1172 & & Hampden \\
\hline Protoperidinium sp. A* & Proto_spA & $?$ & $?$ & $\mathrm{p}$ & & 1172 & & \\
\hline Pyxidinopsis delicata & Pyx_del & $\mathrm{e}$ & $\mathrm{e}$ & $\mathrm{g}$ & & 1172 & & Hampden \\
\hline Pyxidinopsis sp. A* & Pyx_spA & $?$ & $?$ & $\mathrm{~g}$ & & 1172 & & \\
\hline Pyxidinopsis waipawaensis & Pyx_wai & $\mathrm{e}$ & $\mathrm{e}$ & $\mathrm{g}$ & & 1172 & & \\
\hline $\begin{array}{l}\text { Reticulatosphaera } \\
\text { actinocoronata }\end{array}$ & Ret_act & $\mathrm{c}$ & $\mathrm{c}$ & $\mathrm{g}$ & & 1172 & & \\
\hline $\begin{array}{l}\text { Rhombodinium } \\
\text { rhomboideum }\end{array}$ & Rho_rho & wetz & $\mathrm{c}$ & $\mathrm{p}$ & & & & Hampden \\
\hline Rhombodinium spp. & Rho_spp & wetz & $\mathrm{c}$ & $\mathrm{p}$ & & 1172 & & \\
\hline Samlandia septata & Sam_del & $\mathrm{m}$ & $\mathrm{m}$ & $\mathrm{g}$ & & 1172 & & Hampden \\
\hline Schematophora speciosa & Sche_spec & $\mathrm{m}$ & $\mathrm{m}$ & $\mathrm{g}$ & & 1172 & & \\
\hline Selenopemphix spp. & Sel_spp. & $\mathrm{c}$ & $\mathrm{c}$ & $\mathrm{p}$ & 1170 & 1172 & & \\
\hline Senegalinium sp. A brown & Sen_spA & $\mathrm{c}$ & $\mathrm{c}$ & $\mathrm{p}$ & & 1172 & & \\
\hline $\begin{array}{l}\text { Senegalinium sp. B } \\
\text { transparent }\end{array}$ & Sen_spB & $\mathrm{c}$ & $\mathrm{c}$ & $\mathrm{p}$ & & 1172 & & \\
\hline
\end{tabular}




\begin{tabular}{|c|c|c|c|c|c|c|c|c|}
\hline Senegalinium spp. (pars) & Sen_spp & $\mathrm{c}$ & $\mathrm{c}$ & $\mathrm{p}$ & & 1172 & Latrobe & Hampden \\
\hline Spinidinium colemanii & Spi_col & $\mathrm{m}$ & $\mathrm{m}$ & $\mathrm{p}$ & 1170 & & & \\
\hline Spinidinium macmurdoense & Spi_mac & $\mathrm{b}$ & $\mathrm{b}$ & $\mathrm{p}$ & 1170 & 1172 & & Hampden \\
\hline Spinidinium schellenbergii & Spi_sch & $\mathrm{tf}$ & tf & $\mathrm{p}$ & 1170 & 1172 & & Hampden \\
\hline $\begin{array}{l}\text { Spiniferites pseudofurcatus- } \\
\text { type }\end{array}$ & Spi_pseu & $\mathrm{c}$ & $\mathrm{c}$ & $\mathrm{g}$ & & & Latrobe & \\
\hline Spiniferites ramosus & Spinif_ram & $\mathrm{c}$ & $\mathrm{c}$ & $\mathrm{g}$ & & 1172 & & \\
\hline $\begin{array}{l}\text { Spiniferites sp. B cf. } \\
\text { Brinkhuis } 2003\end{array}$ & Spinif_spB & $\mathrm{c}$ & $\mathrm{c}$ & $\mathrm{g}$ & & 1172 & & \\
\hline Spiniferites spp. (pars) & Spinif_spp & $\mathrm{c}$ & $\mathrm{c}$ & $\mathrm{g}$ & 1170 & 1172 & Latrobe & Hampden \\
\hline Stoveracysta kakanuensis & Sto_kak & $\mathrm{e}$ & $\mathrm{e}$ & $\mathrm{g}$ & & 1172 & & \\
\hline Stoveracysta ornata & Sto_orn & $\mathrm{c}$ & $\mathrm{c}$ & $\mathrm{g}$ & & 1172 & & \\
\hline Stoveracysta spp. (pars) & Sto_evi & $\mathrm{c}$ & $\mathrm{c}$ & $\mathrm{g}$ & & & & Hampden \\
\hline Tectatodinium pellitum & Tec_pel & $\mathrm{m}$ & $\mathrm{m}$ & $\mathrm{g}$ & 1170 & & Latrobe & \\
\hline Thalassiphora pelagica & Tha_pel & $\mathrm{c}$ & $\mathrm{c}$ & $\mathrm{g}$ & 1170 & 1172 & & \\
\hline Thalassiphora spp. (pars) & Tha_spp & $\mathrm{c}$ & $\mathrm{c}$ & $\mathrm{g}$ & & & Latrobe & \\
\hline Turbiosphaera filosa & Turb_fil & $\mathrm{m}$ & $\mathrm{m}$ & $\mathrm{g}$ & & 1172 & & \\
\hline Vozzhennikovia apertura & Voz_ape & $\mathrm{b}$ & $\mathrm{b}$ & $\mathrm{p}$ & 1170 & 1172 & & Hampden \\
\hline Vozzhennikovia netrona & Voz_net & tf & tf & $\mathrm{p}$ & 1170 & 1172 & & \\
\hline Vozzhennikovia spp. (pars) & Voz_spp & $\mathrm{b}$ & $\mathrm{b}$ & $\mathrm{p}$ & 1170 & & & \\
\hline Vozzhennikovia stickleyae & Voz_sti & $\mathrm{tf}$ & tf & $\mathrm{p}$ & 1170 & 1172 & & \\
\hline Wetzellioid indet. & Wetz_spp & wetz & $\mathrm{c}$ & $\mathrm{p}$ & & & Latrobe & \\
\hline $\begin{array}{l}\text { Wilsonidium } \\
\text { echinosuturatum** }\end{array}$ & Wil_ech & wetz & $\mathrm{c}$ & $\mathrm{p}$ & & 1172 & & Hampden \\
\hline Wilsonidium ornatum & Wil_orn & wetz & $\mathrm{m}$ & $\mathrm{p}$ & & 1172 & & \\
\hline Dinocyst indet. & Dino_indet & o & o & $\mathrm{o}$ & 1170 & & & \\
\hline Dinocyst G-cyst indet. & Gcyst_indet & o & o & $\mathrm{g}$ & & & Latrobe & \\
\hline Dinocyst P-cyst indet. & Pcyst_indet & o & o & $\mathrm{p}$ & & & & \\
\hline
\end{tabular}

* uncertain biogeographic affinity

** based on in prep. dinocyst results from IODP Site U1408 and U1410 